\title{
Two Variants in the NOTCH4 and HLA-C Genes Contribute to Familial Clustering of Psoriasis
}

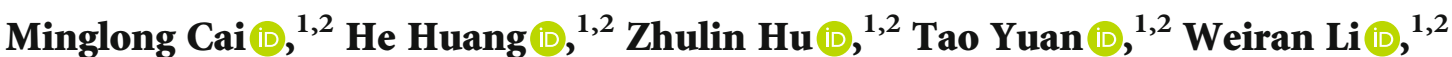 \\ Yaoguang Liu $\mathbb{D}^{1,2}$ Lijun Zheng $\mathbb{D}^{1,2}$ Yan Zhang $\mathbb{D}^{1,2}$ Yujun Sheng $\mathbb{D}^{1,2}$ \\ and Xuejun Zhang $\mathbb{D}^{1,2}$ \\ ${ }^{1}$ Department of Dermatology, The First Affiliated Hospital of Anhui Medical University, Hefei 230032, China \\ ${ }^{2}$ Institute of Dermatology, Anhui Medical University, Hefei 230032, China
}

Correspondence should be addressed to Yujun Sheng; ahmusyj@163.com and Xuejun Zhang; ayzxj@vip.sina.com

Received 24 April 2020; Revised 18 September 2020; Accepted 5 October 2020; Published 20 October 2020

Academic Editor: Julio Martin Garcia

Copyright (C) 2020 Minglong Cai et al. This is an open access article distributed under the Creative Commons Attribution License, which permits unrestricted use, distribution, and reproduction in any medium, provided the original work is properly cited.

\begin{abstract}
Psoriasis is a multifactorial immune-mediated skin disease with a strong genetic background. Previous studies reported that psoriasis with a family history ( $\mathrm{PFH}$ ) and sporadic psoriasis (SP) have a distinct manifestation and genetic predisposition. However, the genetic heterogeneity of PFH and SP in the major histocompatibility complex (MHC) region has not been fully elucidated. To explore genetic variants in the MHC region that drive family aggregation of psoriasis, we included a total of 8,127 psoriasis cases and 9,906 healthy controls from Han Chinese and divided psoriasis into two subtypes, PFH $(n=1,538)$ and SP $(n=5,262)$. Then, we calculated the heritability of PFH and SP and performed a large-scale stratified association analysis. We confirmed that variants in the MHC region collectively explained a higher heritability of PFH (16.8\%) than SP (13.3\%). Further stratified association analysis illustrated that HLA-C * 06:02 and NOTCH4:G511S contribute to the family aggregation of psoriasis, and BTNL2:R281K specifically confers risk for SP. HLA-C * 06:02 and NOTCH4:G511S could partially explain why patients with $\mathrm{PFH}$ have a stronger genetic predisposition, more complex phenotypes, and more frequent other autoimmune diseases. The identification of the SP-specific variant BTNL2:R281K revealed that the genetic architecture of SP is not just a subset of PFH.
\end{abstract}

\section{Introduction}

Psoriasis is a chronic and multifactorial immune-mediated skin disease characterized by epidermal hyperplasia. It affects $0.09 \%-11.4 \%$ of worldwide population $[1,2]$. Psoriasis has a strong genetic predisposition. Evidence shows monozygotic twins have higher concordance rates $(0.2-0.73)$ than dizygotic twins $(0.09-0.2)$ [3-8]. Moreover, $23.1 \%-31.9 \%$ of psoriasis patients have a family history $[9,10]$. To date, linkage analysis and genome-wide association studies (GWAS) have identified more than 80 disease-associated loci, with the most prominent risk loci observed in the human leukocyte antigen (HLA) region [11-14].

Although patients with psoriasis tend to cluster in families, most of them are sporadic. Evidence has shown psoriasis with a family history (PFH) is different from sporadic psoriasis (SP) in terms of both genetic background and phenotype. Genes outside of the major histocompatibility complex (MHC) region such as ERAP1 and NFKB1 were found to be associated with the risk of familial aggregation of psoriasis $[15,16]$. A clinical investigation reported that PFH patients have an earlier onset of psoriasis and more frequently nail disease, enthesitis, and other autoimmune diseases [10, 17]. It seems that PFH has a stronger genetic background and more complicated phenotypes than SP.

However, the effects of the genetic heterogeneity of the HLA alleles on PFH and SP had been largely underexplored in spite of the strong associations between HLA alleles and psoriasis. One of the greatest challenges is that HLA genes are highly polymorphic and can be in strong linkage disequi- 
librium, which has complicated the determination of the independent risk signals. In this study, we devised an analytical approach to identify the heterogeneous effects of HLA alleles on PFH and SP by deep sequencing of the MHC region. We first calculated the heritability of $\mathrm{PFH}$ and SP and confirmed that variants in the MHC region collectively explained a higher heritability of PFH than SP. Then, we considered two hypothetical models of the genetic structure in PFH versus SP: (1) We considered that shared variants might be associated with both PFH and SP; such variants would confer more risk of PFH than of SP. (2) We considered that specific variants might specifically confer risk for $\mathrm{PFH}$; such variants would not be associated with SP. Based on two hypothetical models, we conducted a step-wise association analysis of HLA with PFH and SP to identify those shared and specific variants for psoriasis subtypes. Finally, we performed a case-case analysis (PFH vs. SP) to determine whether those shared and specific variants contribute to the family aggregation of psoriasis.

\section{Materials and Methods}

2.1. Participants. We included a total of 8,127 patients with psoriasis (patients with psoriasis arthritis were not included) and 9,906 geographically matched healthy controls of Chinese ancestry, as previously described [18]. All cases were diagnosed by at least two dermatologists, and all controls were healthy individuals without psoriasis, any autoimmune diseases, or a family history of genetic diseases (including first-, second- and third-degree relatives). Clinical information for all individuals was gathered using a previously developed structured questionnaire. All participants signed informed consent. The study was approved by the institutional ethical committee of the First Affiliated Hospital of Anhui Medical University (No.20131349) and was conducted in accordance with the Declaration of Helsinki principles.

2.2. Sequencing and Quality Control. The samples enrolled for our analysis underwent targeted sequencing and stringent quality control as described in our previous study [18]. The raw sequencing data from samples have been deposited in the Sequence Read Archive (SRA) under the accession number SRA205317. Furthermore, we removed variants with a call rate $<99 \%$, MAF $<0.5 \%$, or Hardy-Weinberg equilibrium of $P<10^{-4}$. A total of 26,775 variants remained for the following analysis.

2.3. Phenotype Classification. We classified psoriasis $(n=8,127)$ into two subtypes, PFH $(n=1,722)$ and SP $(n=6,405)$. To rule out the influence of the confounder of the age of onset, we removed patients with age of onset after 40 years, retaining 1,538 $\mathrm{PFH}$ and 5,262 SP for the following analysis.

2.4. Statistical Analyses. The association of age of onset with a family history of psoriasis was evaluated by the Pearson chisquare test and Student's $t$ test. The association analysis of HLA with psoriasis subtypes was performed using logistic regression, assuming an additive effect of the allele dosages in the log-odds scale. We included sex and region as covari- ates to adjust for population stratification. Association analysis was conducted as follows: (1) association analysis of variants with $\mathrm{PFH}$ and (2) association analysis of variants with SP. For conditional association analysis, we consecutively included the detected variants as covariates to search for additional variants with independent association. This was repeated in a forward stepwise approach until no marker satisfied the significance threshold (Bonferroni correction: $\left.0.05 / 26,775=1.87 \times 10^{-6}\right)$. For those variants that were independently associated with $\mathrm{PFH}$ or SP, we further conducted a case-case association analysis by using logistic regression after adjusting for sex and region. For epistasis analysis, we defined a logistic regression model that simultaneously included interaction terms between significant variants. Association analysis and conditional association analysis were conducted by PLINK (v.1.9) [19]. Plots of HLA associations were drawn by $\mathrm{R}$ (v.3.6.1).

2.5. Annotation of Variants. For variants with amino acid changes, we used SIFT [20], PolyPhen-2 [21], CADD [22], and DANN [23] to measure whether an amino acid is deleterious based on changes in the structure/function of proteins, allelic diversity, pathogenicity, and conservation. The criteria used are as follows: SIFT: deleterious (sift_score $\leq 0.05$ ), tolerated (sift_score $>0.05$ ); PolyPhen-2: probably damaging (pp2 hdiv $\geq 0.957$ ), possibly damaging

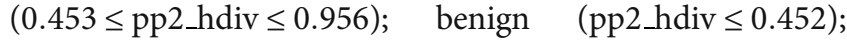
CADD: top $10 \%$ most deleterious (scaled C - scores $\geq 10$ ); top $1 \%$ most deleterious (scaled $\mathrm{C}-$ scores $\geq 20$ ); DANN: top $10 \%$ most deleterious (DANN_score $\geq 0.9$ ); top $1 \%$ most deleterious (DANN_socre $\geq 0.99$ ).

\section{Results}

3.1. Sample Selection and Phenotype Classification. A total of 8,127 psoriasis patients were included. Clinically, psoriasis can be classified into type 1 psoriasis (defined as age of onset before 40) and type 2 psoriasis (defined as age of onset after 40) [24]. It is well known that genetic factors are more prevalent in type 1 psoriasis, and type 1 psoriasis is more likely to be familial [24]. In our study, we observed that type 1 psoriasis was strongly associated with familial aggregation $\left(\mathrm{OR}=1.816, P=9.58 \times 10^{-13}\right.$; Table 1$)$. In order to rule out the influence of age of onset, we excluded patients with age of onset after 40, retaining 1,538 PFH (mean age of onset: $21.79 \pm 9.19$ ) and 5,262 SP (mean age of onset: $22.26 \pm 6.97$ ) cases for the following analysis. There was no substantial correlation between age of onset and family history $(P=0.061)$ after removing patients with type 2 psoriasis.

3.2. Variants Identification. Our target sequencing of the entire 5-Mb MHC region, from upstream of $H L A-A$ to downstream of HLA-DPB1, detected a total of 36,300 indels and 224,872 SNPs [18]. Next, we generated accurate genotyping results (i.e., HLA alleles, amino acid polymorphisms, etc.) for genes including the HLA classic genes (i.e., $H L A-A, H L A-B, H L A-C$, etc.) and the non-HLA genes (i.e., NOTCH4, TAP2, MICA, etc.). After stringent quality control as previously described [18], we further removed variants 
TABLE 1: Description of study samples of patients with psoriasis.

\begin{tabular}{lcccccr}
\hline Subtypes & \multicolumn{3}{c}{$\begin{array}{c}\text { Age of onset before removing } \\
\text { type 2 psoriasis }\end{array}$} & \multicolumn{2}{c}{$\begin{array}{c}\text { Age of onset after removing } \\
\text { type 2 psoriasis }\end{array}$} \\
& $\leq 40$ & $>40$ & OR & $P$ & Mean $\pm \mathrm{SD}^{1}$ & $P$ \\
\hline PBH & 1,538 & 184 & 1.82 & $9.58 \times 10^{-13}$ & $21.79 \pm 9.19$ & 0.061 \\
SP & 5,262 & 1,143 & & $22.26 \pm 6.97$ & \\
\hline
\end{tabular}

${ }^{1}$ SD: standard deviation.

with a call rate $<99 \%$, minor allele frequency $(\mathrm{MAF})<0.5 \%$, or Hardy-Weinberg equilibrium of $P<10^{-4}$. A total of 26,775 variants, including HLA alleles, amino acid polymorphisms, SNPs, and short indels remained for the following analysis, with most of them (63.6\%) being common variants (MAF > 5\%).

3.3. Heritability of PFH and SP. To determine whether the genetic background of $\mathrm{PFH}$ is stronger than that of SP, GCTA (v1.93.1) [25] was used to calculate the heritability of PFH and SP under the assumption of a disease prevalence of $0.47 \%$. We observed that variants in the MHC region collectively explained a higher heritability of PFH $\left(h^{2}=16.8 \%\right.$, standard error $=0.0158)$ than SP $\left(h^{2}=13.3 \%\right.$, standard error $=0.0095)$. This confirmed that $\mathrm{PFH}$ has a stronger genetic predisposition than SP in terms of the MHC region.

3.4. Associations of HLA with PFH. We performed a stepwise association analysis of variants with PFH susceptibility (1,538 PFH vs. 9,906 control). The most significantly associated variant mapped to the classical HLA-C * 06:02 allele $\left(\mathrm{OR}=12.92, P=1.00 \times 10^{-352}\right.$; Figure 1, Table 2), followed by $H L A-C * 07: 04\left(\mathrm{OR}=3.19, P=6.63 \times 10^{-10}\right)$. After conditioned on $H L A-C * 06: 02$ and $H L A-C * 07: 04$, there was no variant in $H L A-C$ that reached the predefined threshold $\left(P=1.87 \times 10^{-6}\right)$. To look for additional independent variants, we conditioned on the $H L A-C$ region and observed a novel significant risk variant for NOTCH4:G511S $\left(\mathrm{OR}=2.15, P=7.22 \times 10^{-15}\right)$. When we conditioned on HLA-C and NOTCH4, we observed an independent association at $H L A-B$ amino acid position $67(B-Y 67 C, \mathrm{OR}=1.80$, $\left.P=5.45 \times 10^{-11}\right)$, which was previously reported to be associated with psoriasis in both European and Han Chinese populations $[18,26]$. After conditioning on HLA-C, NOTCH4, and $H L A-B$, we discovered an independent association for $H L A-D P B 1$ amino acid position 35 (DPB1-F35L, OR $=1.38$, $\left.P=1.86 \times 10^{-10}\right) . D P B 1-F 35 L$ was in strong linkage disequilibrium with a previously reported psoriasis-associated allele, HLA-DPB1 * 05:01 $\left(r^{2}=0.76\right)$ [18]. Further stepwise analysis identified another significant allele for TAP2:01:03 $\left(\mathrm{OR}=0.41, P=1.02 \times 10^{-06}\right)$. When we conditioned on all the above genes, no additional variant remained significantly associated with PFH susceptibility.

3.5. Associations of HLA with SP. As expected, in the finemapping analysis of SP (5,262 SP cases vs. 9,906 controls), we observed HLA-C * 06:02 ( $\mathrm{OR}=15.16, P=1.00 \times 10^{-863}$, Figure 2, Table 3 ) as the most significantly SP-associated allele, followed by HLA-C * 07:04 (OR = 3.89, $\left.P=5.35 \times 10^{-27}\right)$. After the condition on HLA-C *06:02 and HLA-C *07:04, we found an independent association for HLA-C * 01:02 $\left(\mathrm{OR}=1.29, P=8.35 \times 10^{-8}\right)$, which was recently reported to be associated with psoriasis in southern China [27]. We then investigated additional risk variants independent of HLA-C. When we included $H L A-C$ as covariant, we observed significant independent associations at HLA-B amino acids 67 $\left(B-Y 67 C, \mathrm{OR}=2.26, P=2.30 \times 10^{-47}\right), 116(B-Y 116 S, \mathrm{OR}=$ $\left.1.38, P=7.82 \times 10^{-20}\right)$, and $70(B-Q 70 K, \mathrm{OR}=0.46, P=7.71$ $\left.\times 10^{-10}\right)$. When we conditioned on HLA-C and HLA-B, we discovered a significant allele for HLA-DPB1 * 05:01 $\left(\mathrm{OR}=1.29, \quad P=5.51 \times 10^{-15}\right)$. Then, we conditioned on HLA-C, HLA-B, and HLA-DPB1. We observed that HLA-A amino acid $95\left(A-I 95 \mathrm{~V}, \mathrm{OR}=1.27, P=8.99 \times 10^{-11}\right)$ exceeded the significance threshold. Further stepwise conditional analysis found that $B T N L 2: R 281 K\left(\mathrm{OR}=1.46, P=7.18 \times 10^{-9}\right)$ was an independent risk variant. After conditioning on all the above genes, we observed no other significant associations.

3.6. Case-Case Association Analysis. Fine-mapping analysis of PFH and SP has identified a total of 11 independent susceptibility variants. To further determine if these 11 variants contribute to the family aggregation of psoriasis, we conducted a case-case analysis (PFH vs. SP), using a $P$ value of $4.54 \times 10^{-3}$ (Bonferroni correction for 11 variants, $0.05 / 11$ ) as the cutoff for statistical significance. We found that HLA-C* 06:02 $\left(\mathrm{OR}=1.33, \quad P=2.63 \times 10^{-5}\right)$ and NOTCH4:G511S $\left(\mathrm{OR}=1.24, \quad P=4.26 \times 10^{-3}\right)$ confer risk for $\mathrm{PFH}$, while BTNL2:R281K $\left(\mathrm{OR}=0.74, P=2.05 \times 10^{-3}\right)$ confers risk for SP (Table 4).

3.7. Annotation of NOTCH4:G511S and BTNL2:R281K. For variants NOTCH4:G511S and BTNL2:R281K, we used four annotation programs (see Materials and Methods) to measure the deleteriousness of variants. SIFT predicted that NOTCH4:G511S (sift_score $=0.04$ ) and BTNL2:R281K (sift_score $=0.04)$ are deleterious. PolyPhen-2 predicted that $B T N L 2: R 281 \mathrm{~K}$ is probably damaging (pp2_hdiv $=0.999)$, and NOTCH4:G511S is possible damaging (pp2 hdiv $=0.847$ ), respectively. Both CADD and DANN predicted that BTNL2:R281K (scaled C - score $=29.7$ and DANN_score $=$ 0.997 ) and NOTCH4:G511S (scaled C-score $=23.3$ and DANN_score $=0.997$ ) are the top $1 \%$ most deleterious variants.

3.8. No Epistasis between HLA-C * 06:02 and NOTCH4:G511S. Our stratified analysis suggested that NOTCH4:G511S and $H L A-C * 06: 02$ contribute to the familial clustering of psoriasis. To further investigate whether there is an interaction between NOTCH4:G511S and HLA-C * 06:02, we performed an epistasis analysis by including interaction terms in the logistic regression model. In the case-case analysis, we included an 

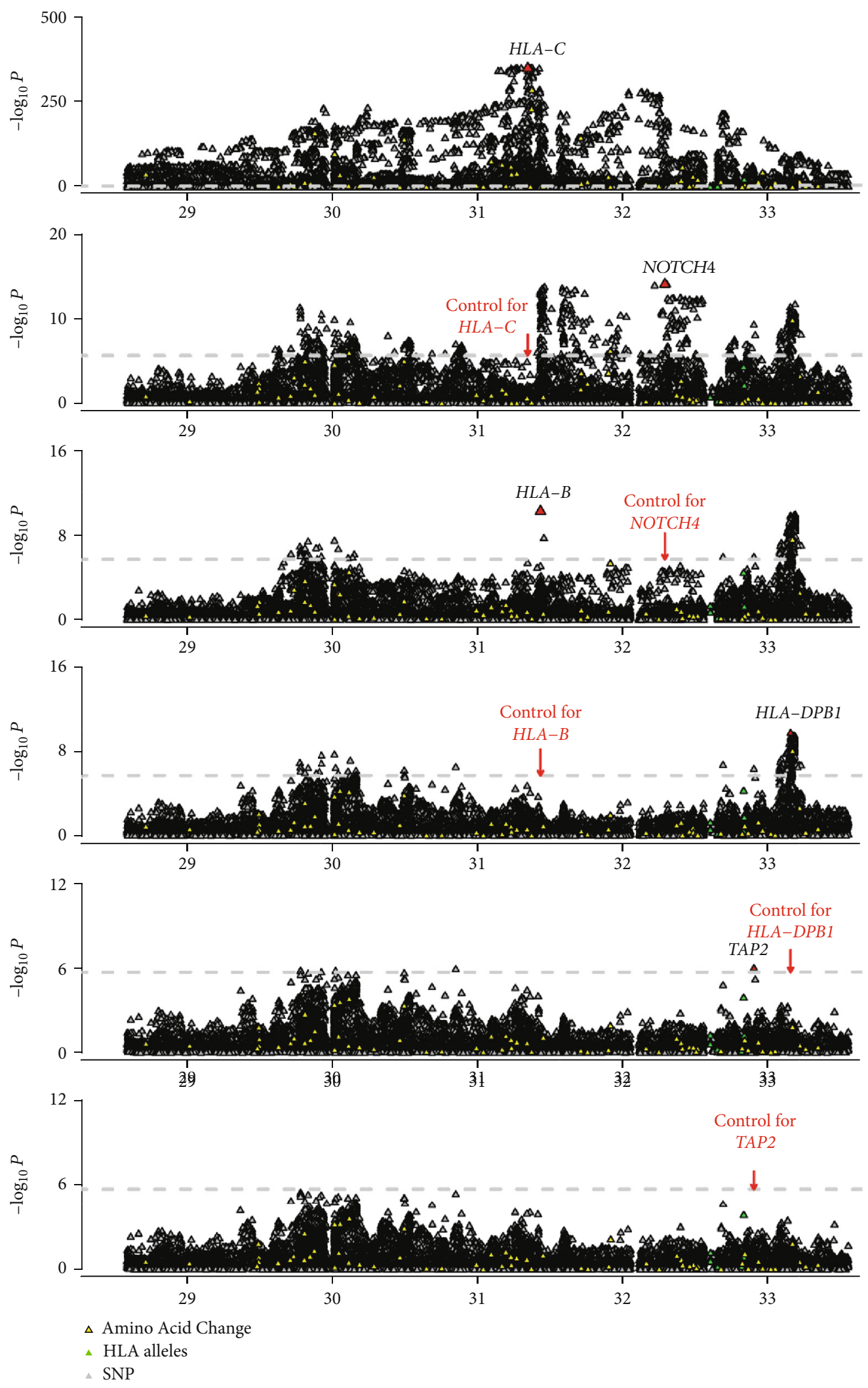

FIGURE 1: A Manhattan plot of the stepwise conditional association for psoriasis with a family history (PFH) in the major histocompatibility complex (MHC) region. Stepwise analysis of HLA-C, NOTCH4, HLA-B, HLA-DPB1, and TAP2 in PFH vs. control. For each plot, the horizontal axis shows the genomic position, and the vertical axis shows negative $\log _{10}$-transformed $P$ values for association. The dashed horizontal line corresponds to the significance threshold of $P=1.87 \times 10^{-6}$. The red triangles represent the top associated variants. 
TABLE 2: Association of variants with psoriasis with a family history (PFH) in the multivariate regression model.

\begin{tabular}{|c|c|c|c|c|}
\hline \multirow{2}{*}{ Variants } & \multicolumn{2}{|c|}{ Frequency } & \multicolumn{2}{|c|}{ PFH vs. control } \\
\hline & Freq_PFH ${ }^{1}$ & Freq_Control $^{2}$ & OR $(95 \% \mathrm{CI})$ & $P$ value \\
\hline \multicolumn{5}{|l|}{ HLA-C alleles } \\
\hline$H L A-C * 06: 02$ & 0.479 & 0.110 & $12.92(11.41-14.64)$ & $1.00 \times 10^{-352}$ \\
\hline$H L A-C * 07: 04$ & 0.015 & 0.010 & $3.19(2.21-4.61)$ & $6.63 \times 10^{-10}$ \\
\hline \multicolumn{5}{|l|}{ NOTCH4 } \\
\hline NOTCH4:G511S & 0.092 & 0.018 & $2.15(1.77-2.61)$ & $7.22 \times 10^{-15}$ \\
\hline \multicolumn{5}{|l|}{ HLA-B alleles } \\
\hline$B-Y 67 C$ & 0.082 & 0.087 & $1.80(1.51-2.15)$ & $5.45 \times 10^{-11}$ \\
\hline \multicolumn{5}{|l|}{ HLA-DPB1 alleles } \\
\hline$D P B 1-F 35 L$ & 0.407 & 0.437 & $1.38(1.25-1.52)$ & $1.86 \times 10^{-10}$ \\
\hline \multicolumn{5}{|l|}{ TAP2 } \\
\hline TAP2 $* 01: 03$ & 0.028 & 0.025 & $0.41(0.29-.59)$ & $1.02 \times 10^{-6}$ \\
\hline
\end{tabular}

${ }^{1}$ Freq_PFH: the allele frequency in patients with PFH. ${ }^{2}$ Freq_Control: the allele frequency in healthy controls.

interaction term between NOTCH4:G511S and HLA-C * 06:02 in our logistic regression model, and we observed no evidence of interaction between NOTCH4:G511S and HLA-C * 06:02 $(P=0.648)$. In the $\mathrm{PFH}$ versus control analysis, 15 interaction terms between all 6 significant variants were included in the logistic regression model; a $P$ value of $0.003(0.05 / 15)$ was considered as statistical significance. We found strong interactions between HLA-C * 06:02 and TAP2 * 01:03 (OR = 0.35, P= $\left.1.7 \times 10^{-9}\right)$ and HLA-C * 06:02 and DPB1-F35L (OR = 1.57, $\left.P=1.7 \times 10^{-6}\right)$. But we still observed no evidence of interaction between HLA-C * 06:02 and NOTCH4:G511S after Bonferroni correction $(\mathrm{OR}=0.55, P=0.007)$. Our results suggested that HLA-C * 06:02 and NOTCH4:G511S may independently contribute to the familial clustering of psoriasis.

\section{Discussion}

In this study, we performed a large-scale stratified analysis to detect HLA and non-HLA variants in the MHC region that drive familial aggregation of psoriasis. In fine-mapping analysis of PFH and SP, we identified a total of 11 independent variants, of which eight variants $(C * 06: 02, C * 07: 04, C *$ 01:02, HLA-B amino acid positions 67, 116, HLA-A amino acid position 95, DPB1 * 05:01, and BTNL2:R281K) were reported to be associated with psoriasis by previous studies [18, 26-28]. Notably, HLA-C * 06:02, HLA-C * 07:04, $H L A-B$ amino acid position 67, and HLA-DPB1 * 05:01 (strong linkage disequilibrium with $D P B 1-F 35 L$ ) were shared susceptibility variants between $\mathrm{PFH}$ and $\mathrm{SP}$, while other variants (NOTCH4:G511S and TAP2 * 01:03 for PFH, C*01:02, $B-Y 116 S, B-Q 70 K, A-I 95 V$, and BTNL2:R281K for SP) showed specific associations with one of two psoriasis subtypes. Here, we have successfully decomposed the genetic architecture of PFH and SP into shared components and specific components. In order to further determine whether these shared or specific variants contribute to the family aggregation of psoriasis, we conducted a case-case analysis (PFH vs. SP) and eventually identified two variants (HLA-C
* 06:02 and NOTCH4:G511S) in the MHC region which confer risk for family aggregation of psoriasis. Moreover, we also identified a variant (BTNL2:R281K) that specifically confers risk for SP.

For those shared variants between PFH and SP, only HLA$C * 06: 02$ was validated to be associated with family aggregation of psoriasis in the case-case analysis $(\mathrm{OR}=1.33, P=$ $\left.2.63 \times 10^{-5}\right)$. HLA-C $* 06: 02$ is a well-known psoriasis-risk allele, and it was reported to be strongly associated with early age of onset of psoriasis [29]. This might explain why PFH have an earlier onset of psoriasis. Therefore, $H L A-C * 06: 02$ conforms to our first hypothetical model that the shared variant contributes more risk to PFH than to SP. For those specific variants in PFH or SP, we found that NOTCH4:G511S $\left(\mathrm{OR}=1.24, P=4.26 \times 10^{-3}\right)$ contributes to the familial clustering of psoriasis. NOTCH4:G511S was proven to be an independent risk variant for PFH because the effect of NOTCH4:G511S with respect to $\mathrm{PFH}$ vs. control risk remained significant even after we conditioned on HLA-C, HLA-B, HLA-DPB1, HLA-A, TAP2, and BTNL2 $\left(P=1.62 \times 10^{-12}\right.$, Table S1A). In contrast, the impact of NOTCH4:G511S on SP vs. control risk disappeared after we conditioned on $H L A-C$ and $H L A-B$ $\left(P>1.87 \times 10^{-6}\right)$. Therefore, NOTCH4:G511S conforms to the second hypothetical model that the specific variant only confers risk for $\mathrm{PFH}$ and not for SP. NOTCH4 encodes members of the notch family of proteins that positively regulate notch signaling. The notch signaling pathway is a highly conserved molecular network that has a key role in angiogenesis [30], cellular proliferation and differentiation [31], and apoptosis [32]. Other studies have reported that the notch pathway regulates several critical aspects of epidermal renewal and development, including proliferation, differentiation, and cell-fate of keratinocytes [33-36]. For example, a previous study showed that notch signaling induces differentiation of spinous cells into granulosa cells and, simultaneously, prevents premature differentiation of spinous cells, orchestrating the balance between differentiation and immature programs in suprabasal 

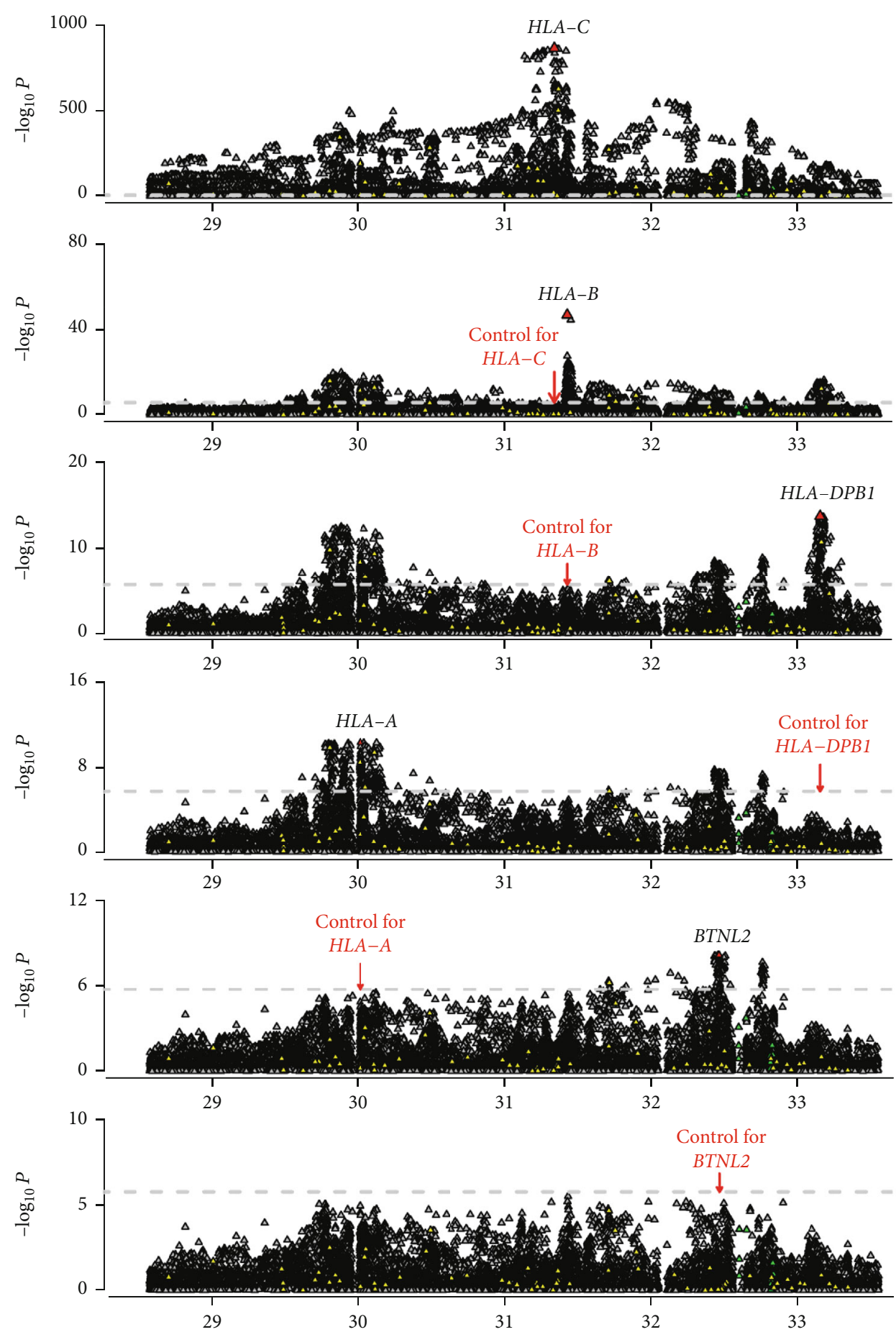

$\triangle$ Amino acid change

$\triangle$ HLA alleles

$\triangle \mathrm{SNP}$

Figure 2: A Manhattan plot of the stepwise conditional association for SP in the MHC region. Stepwise analysis of $H L A-C, H L A-B$, $H L A-D P B 1, H L A-A$, and BTNL2 in SP vs. control. For each plot, the horizontal axis shows the genomic position and the vertical axis shows the negative $\log _{10}$-transformed $P$ values for the association. The dashed horizontal line corresponds to the significance threshold of $P=1.87 \times 10^{-6}$. The red triangles represent the top associated variants.

cells during epidermal development [36]. Moreover, the amino acid substitution glycine $>$ serine at position 511 in NOTCH4 was predicted to be deleterious by all four annotation tools, suggesting this variant (NOTCH4:G511S) may cause aberrations in notch signaling and further lead to aggressive angiogenesis and the disruption of keratinocyte differentiation in psoriasis lesions. In addition, previous studies reported that $\mathrm{NOTCH} 4$ was associated with multiple 
TABLE 3: Association of variants with sporadic psoriasis (SP) in the multivariate regression model.

\begin{tabular}{|c|c|c|c|c|}
\hline \multirow{2}{*}{ Variants } & \multicolumn{2}{|c|}{ Frequency } & \multicolumn{2}{|c|}{ SP vs. control } \\
\hline & Freq_SP ${ }^{1}$ & Freq_Control $^{2}$ & OR $(95 \% \mathrm{CI})$ & $P$ value \\
\hline \multicolumn{5}{|l|}{ HLA-C alleles } \\
\hline HLA-C $* 06: 02$ & 0.444 & 0.110 & $15.16(13.93-16.49)$ & $1.00 \times 10^{-863}$ \\
\hline HLA-C $* 07: 04$ & 0.0148 & 0.010 & $3.89(3.04-4.99)$ & $5.35 \times 10^{-27}$ \\
\hline HLA-C $* 01: 02$ & 0.107 & 0.139 & $1.29(1.18-1.42)$ & $8.35 \times 10^{-08}$ \\
\hline \multicolumn{5}{|l|}{ HLA-B alleles } \\
\hline B-Y67C & 0.093 & 0.087 & $2.26(2.03-2.53)$ & $2.30 \times 10^{-47}$ \\
\hline B-Y116S & 0.316 & 0.326 & $1.38(1.29-1.48)$ & $7.82 \times 10^{-20}$ \\
\hline B-Q70K & 0.016 & 0.023 & $0.46(0.36-0.59)$ & $7.71 \times 10^{-10}$ \\
\hline \multicolumn{5}{|l|}{ HLA-DPB1 alleles } \\
\hline $\mathrm{DPB} 1 * 05: 01$ & 0.362 & 0.364 & $1.29(1.21-1.38)$ & $5.51 \times 10^{-15}$ \\
\hline \multicolumn{5}{|l|}{ HLA-A alleles } \\
\hline A-I95V & 0.257 & 0.299 & $1.27(1.18-1.37)$ & $8.89 \times 10^{-11}$ \\
\hline \multicolumn{5}{|l|}{ BTNL2 } \\
\hline BTNL2:R281K & 0.059 & 0.063 & $1.46(1.29-1.66)$ & $7.18 \times 10^{-9}$ \\
\hline
\end{tabular}

${ }^{1}$ Freq_SP: the allele frequency in patients with SP. ${ }^{2}$ Freq_Control: the allele frequency in healthy controls.

TABLE 4: Association of human leukocyte antigen (HLA) with a family history of psoriasis.

\begin{tabular}{|c|c|c|c|c|}
\hline \multirow{2}{*}{ Variants } & \multicolumn{2}{|c|}{ Frequency } & \multicolumn{2}{|c|}{ PFH vs. SP } \\
\hline & Freq_PFH & Freq_SP & OR $(95 \% \mathrm{CI})$ & $P$ value \\
\hline$H L A-C * 06: 02^{1}$ & 0.479 & 0.444 & $1.33(1.17-1.52)$ & $2.63 \times 10^{-5}$ \\
\hline HLA-C $* 07: 04$ & 0.015 & 0.015 & $0.92(0.65-1.29)$ & 0.624 \\
\hline HLA-C $* 01: 02$ & 0.092 & 0.107 & $0.93(0.80-1.07)$ & 0.295 \\
\hline B-Y67C & 0.081 & 0.092 & $0.86(0.74-0.998)$ & 0.047 \\
\hline B-Y116S & 0.313 & 0.316 & $1.01(0.92-1.1)$ & 0.920 \\
\hline B-Q70K & 0.016 & 0.016 & $1.03(0.74-1.44)$ & 0.855 \\
\hline $\mathrm{DPB} 1 * 05: 01$ & 0.354 & 0.362 & $0.98(0.89-1.07)$ & 0.591 \\
\hline A-I95V & 0.250 & 0.257 & $0.95(0.86-1.05)$ & 0.287 \\
\hline NOTCH4:G511S & 0.092 & 0.076 & $1.24(1.07-1.44)$ & $4.26 \times 10^{-3}$ \\
\hline BTNL2:R281K $K^{1}$ & 0.044 & 0.059 & $0.74(0.61-0.89)$ & $2.05 \times 10^{-3}$ \\
\hline TAP $2 * 01: 03$ & 0.028 & 0.026 & $1.04(0.81-1.35)$ & 0.76 \\
\hline
\end{tabular}

${ }^{1}$ Variants with $P<4.54 \times 10^{-3}$ in the significant threshold in case-case analysis and are marked in italics.

autoimmune diseases, including Crohn's disease, systemic scleroderma, rheumatoid arthritis, and ulcerative colitis [37-40]. Of note, enthesitis is a key early manifestation of psoriasis arthritis. NOTCH4 was also reported to be associated with psoriasis arthritis [41], and notch signaling pathways were found to mediate synovial angiogenesis in psoriatic arthritis [42]. These findings suggested that notch signaling pathways were involved in the pathogenesis of $\mathrm{PFH}$ and NOTCH4:G511S may partially explain why PFH has more frequent enthesitis and other autoimmune diseases. We also conducted an epistasis analysis, finding no evidence of interaction between NOTCH4:G511S and HLA-C * 06:02. Therefore, NOTCH4:G511S and HLA-C * 06:02 may independently contribute to the family clustering of psoriasis based on our two hypothetical models.
Besides, we identified a variant (BTNL2:R281K) that specifically confers risk for SP. The amino acid substitution arginine > lysine at position 281 in BTNL2 was predicted to be deleterious by all four annotation tools. Notably, this variant was not associated with either PFH $\left(P=4.29 \times 10^{-5}\right)$ or SP $(P=0.19)$ in the univariate analysis (Table S1A). However, it exhibited a significant association with SP after conditioning on HLA-C *06:02 $\left(P=6.8 \times 10^{-7}\right)$. Subsequently, we classified SP and control into two subgroups based on the presence of $H L A-C * 06: 02$, the C* 06:02-positive group and the $C * 06: 02$-negative group. We found that although the raw frequency of BTNL2:R281K in SP (0.059) was lower than in controls (0.063), this was reversed in both subgroups (0.086 in SP and 0.070 in controls for the C*06:02-positive group, and 0.054 in SP and 
0.036 in controls for $C * 06: 02$-negative group, Table S1B). Moreover, univariate analysis in these two subgroups showed a consistent effect $(P=0.01$ for the $C * 06: 02$-negative group, $P=1.52 \times 10^{-5}$ for the $\mathrm{C} * 06: 02$-positive group). This phenomenon can be explained by Simpson's paradox [43], where two subgroups have the same association, but the overall population shows no association. In contrast, BTNL2:R281K did not reach the significance threshold in $\mathrm{PFH}$ vs. control after conditioning on other susceptibility variants. The case-case analysis also showed that the frequency of $B T N L 2: R 281 K$ was significantly higher in SP than in PFH $\left(P=2.05 \times 10^{-3}\right)$. Therefore, BTNL2:R281K is an SP-specific risk variant that does not conform to our hypothetical models, which suggests that the genetic architecture of SP is not just a subset of PFH despite the fact that $\mathrm{PFH}$ has a stronger genetic predisposition than SP. We speculate that BTNL2:R281K might be associated with certain phenotypes of SP. For example, a recent study found that patients with SP have a higher Body Mass Index (BMI) than those with PFH [44]. BTNL2 was reported to be associated with the waist-hip ratio [45] and might contribute to higher $\mathrm{BMI}$ in SP.

However, there are limitations to our study. We were unable to perform further genotype-phenotype analysis because the included samples lacked clinical information, such as BMI, enthesitis, and other phenotypes of psoriasis. Besides, the functional mechanisms by which these variants contribute to familial aggregation of psoriasis or disease phenotypes remain unclear. Further studies will be required to address these problems.

In conclusion, we have identified two variants that independently contribute to family clustering of psoriasis, and one variant specifically confers risk for SP. We found NOTCH4:G511S and HLA-C * 06:02 could partially explain why patients with a family history have an earlier onset of psoriasis and more frequent enthesitis and other autoimmune diseases. Our findings support the hypothetical model that PFH has a stronger genetic background than SP, but also indicate that the genetic architecture of SP is not just a subset of PFH. Three variants identified in our study enable to differentiate PFH from SP and might serve as markers to predict the risk of developing psoriasis for individuals at a young age who have a family history.

\section{Data Availability}

The raw sequencing data from samples have been deposited in the Sequence Read Archive (SRA) under the accession SRA205317 (http://www.ncbi.nlm.nih.gov/sra/).

\section{Conflicts of Interest}

The author(s) declare(s) that there is no conflict of interest regarding the publication of this paper.

\section{Acknowledgments}

We are grateful to all participants, their families, and healthy controls who donated blood samples in this study. We also thank LetPub (http://www.letpub.com) for its linguistic assistance during the preparation of this manuscript. This research was funded by the National Natural Science Foundation of China $(81872527,81130031)$.

\section{Supplementary Materials}

Table S1A: Detailed stepwise association results of NOTCH4:G534S and BTNL2:R281K with psoriasis risk. Table S1B: Phenomenon of Simpson's paradox in the association of BTNL2:R281K with SP. (Supplementary Materials)

\section{References}

[1] S. Gibbs, "Skin disease and socioeconomic conditions in rural Africa: Tanzania," International Journal of Dermatology, vol. 35, no. 9, pp. 633-639, 1996.

[2] K. Danielsen, A. O. Olsen, T. Wilsgaard, and A. S. Furberg, "Is the prevalence of psoriasis increasing? A 30-year follow-up of a population-based cohort," The British Journal of Dermatology, vol. 168, no. 6, pp. 1303-1310, 2013.

[3] E. M. Farber, M. L. Nall, and W. Watson, "Natural history of psoriasis in 61 twin pairs," Archives of Dermatology, vol. 109, no. 2, pp. 207-211, 1974.

[4] F. Brandrup, M. Hauge, K. Henningsen, and B. Eriksen, "Psoriasis in an unselected series of twins," Archives of Dermatology, vol. 114, no. 6, pp. 874-878, 1978.

[5] F. Brandrup, N. Holm, N. Grunnet, K. Henningsen, and H. E. Hansen, "Psoriasis in monozygotic twins: variations in expression in individuals with identical genetic constitution," Acta Dermato-Venereologica, vol. 62, no. 3, pp. 229-236, 1982.

[6] D. L. Duffy, L. S. Spelman, and N. G. Martin, "Psoriasis in Australian twins," Journal of the American Academy of Dermatology, vol. 29, no. 3, pp. 428-434, 1993.

[7] A. M. Grjibovski, A. O. Olsen, P. Magnus, and J. R. Harris, "Psoriasis in Norwegian twins: contribution of genetic and environmental effects," Journal of the European Academy of Dermatology and Venereology, vol. 21, no. 10, pp. 1337-1343, 2007.

[8] A. S. Lonnberg, L. Skov, A. Skytthe, K. O. Kyvik, O. B. Pedersen, and S. F. Thomsen, "Heritability of psoriasis in a large twin sample," The British Journal of Dermatology, vol. 169, no. 2, pp. 412-416, 2013.

[9] K. Chen, G. Wang, H. Jin et al., "Clinic characteristics of psoriasis in China: a nationwide survey in over 12000 patients," Oncotarget, vol. 8, no. 28, pp. 46381-46389, 2017.

[10] D. Solmaz, S. Bakirci, G. Kimyon et al., "The impact of having family history of psoriasis or psoriatic arthritis on psoriatic disease," Arthritis Care \& Research, vol. 1, no. 2, p. 3, 2019.

[11] F. Capon, "The Genetic Basis of Psoriasis," International Journal of Molecular Sciences, vol. 18, no. 12, p. 2526, 2017.

[12] X. Fan, S. Yang, W. Huang et al., "Fine mapping of the psoriasis susceptibility locus PSORS1 supports HLA-C as the susceptibility gene in the Han Chinese population," PLoS Genetics, vol. 4, no. 3, article e1000038, 2008.

[13] X.-J. Zhang, W. Huang, S. Yang et al., "Psoriasis genome-wide association study identifies susceptibility variants within LCE gene cluster at 1q21," Nature Genetics, vol. 41, no. 2, pp. 205-210, 2009.

[14] Genetic Analysis of Psoriasis Consortium \& the Wellcome Trust Case Control Consortium 2, A. Strange, F. Capon 
et al., "A Genome-wide association study identifies new psoriasis susceptibility loci and an interaction between HLA-C and ERAP1," Nature Genetics, vol. 42, no. 11, pp. 985-990, 2010.

[15] W. Wang, Z. Zhu, C. Zhu et al., "A genetic variant rs1020760at NFKB1 is associated with clinical features of psoriasis vulgaris in a Han Chinese population," Annals of Human Genetics, vol. 80, no. 4, pp. 197-202, 2016.

[16] Y. Fu, X. Li, Y. Chen, R. Liu, R. Wang, and N. Bai, “Association of ERAP1 gene polymorphisms with the susceptibility to psoriasis vulgaris: a case-control study," Medicine, vol. 97, no. 41, article e12828, 2018.

[17] Y. H. Huang, C. F. Kuo, L. H. Huang, and M. Y. Hsieh, "Familial aggregation of psoriasis and co-aggregation of autoimmune diseases in affected families," Journal of Clinical Medicine, vol. 8, no. 1, p. 115, 2019.

[18] F. Zhou, H. Cao, X. Zuo et al., "Deep sequencing of the MHC region in the Chinese population contributes to studies of complex disease," Nature Genetics, vol. 48, no. 7, pp. 740746, 2016.

[19] C. C. Chang, C. C. Chow, L. C. A. M. Tellier, S. Vattikuti, S. M. Purcell, and J. J. Lee, "Second-generation PLINK: rising to the challenge of larger and richer datasets," Gigascience, vol. 4, no. 1, p. 7, 2015.

[20] R. Vaser, S. Adusumalli, S. N. Leng, M. Sikic, and P. C. Ng, "SIFT missense predictions for genomes," Nature Protocols, vol. 11, no. 1, pp. 1-9, 2016.

[21] I. A. Adzhubei, S. Schmidt, L. Peshkin et al., "A method and server for predicting damaging missense mutations," Nature Methods, vol. 7, no. 4, pp. 248-249, 2010.

[22] M. Kircher, D. M. Witten, P. Jain, B. J. O'Roak, G. M. Cooper, and J. Shendure, "A general framework for estimating the relative pathogenicity of human genetic variants," Nature Genetics, vol. 46, no. 3, pp. 310-315, 2014.

[23] D. Quang, Y. Chen, and X. Xie, "DANN: a deep learning approach for annotating the pathogenicity of genetic variants," Bioinformatics, vol. 31, no. 5, pp. 761-763, 2015.

[24] T. Henseler and E. Christophers, "Psoriasis of early and late onset: characterization of two types of psoriasis vulgaris," Journal of the American Academy of Dermatology, vol. 13, no. 3, pp. 450-456, 1985.

[25] J. Yang, S. H. Lee, M. E. Goddard, and P. M. Visscher, "GCTA: a tool for genome-wide complex trait analysis," American Journal of Human Genetics, vol. 88, no. 1, pp. 76-82, 2011.

[26] Y. Okada and B. Han, "Fine mapping major histocompatibility complex associations in psoriasis and its clinical subtypes," The American Journal of Human Genetics, vol. 95, no. 2, pp. 162-172, 2014.

[27] M. Cai, H. Huang, D. Ran et al., "HLA-C*01:02 and HLA-A* 02:07 confer risk specific for psoriatic patients in southern China," Journal of Investigative Dermatology, vol. 139, no. 9, pp. 2045-2048.e4, 2019.

[28] J. Hirata, T. Hirota, T. Ozeki et al., "Variants at HLA-A, HLA$\mathrm{C}$, and HLA-DQB1 confer risk of psoriasis vulgaris in Japanese," The Journal of Investigative Dermatology, vol. 138, no. 3, pp. 542-548, 2018.

[29] A. Wisniewski, L. Matusiak, A. Szczerkowska-Dobosz, I. Nowak, and P. Kusnierczyk, "HLA-C $* 06: 02$-independent, genderrelated association of PSORS1C3 and PSORS1C1/CDSN single-nucleotide polymorphisms with risk and severity of psoriasis," Molecular Genetics and Genomics, vol. 293, no. 4, pp. 957-966, 2018.
[30] T. Iso, Y. Hamamori, and L. Kedes, "Notch signaling in vascular development," Arteriosclerosis, Thrombosis, and Vascular Biology, vol. 23, no. 4, pp. 543-553, 2003.

[31] J. Pancewicz and C. Nicot, "Current views on the role of Notch signaling and the pathogenesis of human leukemia," $B M C$ Cancer, vol. 11, no. 1, p. 502, 2011.

[32] F. MacKenzie, P. Duriez, F. Wong, M. Noseda, and A. Karsan, "Notch4 inhibits endothelial apoptosis via RBP-Jkappadependent and -independent pathways," The Journal of Biological Chemistry, vol. 279, no. 12, pp. 11657-11663, 2004.

[33] D. Massi and J. Panelos, "Notch signaling and the developing skin epidermis," Advances in Experimental Medicine and Biology, vol. 727, pp. 131-141, 2012.

[34] B. J. Nickoloff, J. Z. Qin, V. Chaturvedi, M. F. Denning, B. Bonish, and L. Miele, "Jagged-1 mediated activation of notch signaling induces complete maturation of human keratinocytes through NF-kappaB and PPARgamma," Cell Death and Differentiation, vol. 9, no. 8, pp. 842-855, 2002.

[35] J. Panelos and D. Massi, "Emerging role of Notch signaling in epidermal differentiation and skin cancer," Cancer Biology \& Therapy, vol. 8, no. 21, pp. 1986-1993, 2009.

[36] M. Moriyama, A. D. Durham, H. Moriyama et al., "Multiple roles of Notch signaling in the regulation of epidermal development," Developmental Cell, vol. 14, no. 4, pp. 594-604, 2008.

[37] K. Yamazaki, J. Umeno, A. Takahashi et al., "A genome-wide association study identifies 2 susceptibility Loci for Crohn's disease in a Japanese population," Gastroenterology, vol. 144, no. 4, pp. 781-788, 2013.

[38] O. Gorlova, J. E. Martin, B. Rueda et al., "Identification of novel genetic markers associated with clinical phenotypes of systemic sclerosis through a genome-wide association strategy," PLoS Genetics, vol. 7, no. 7, article e1002178, 2011.

[39] C. Terao, R. Yamada, K. Ohmura et al., "The human AIRE gene at chromosome 21q22 is a genetic determinant for the predisposition to rheumatoid arthritis in Japanese population," Human Molecular Genetics, vol. 20, no. 13, pp. 26802685, 2011.

[40] G. Juyal, S. Negi, A. Sood et al., "Genome-wide association scan in north Indians reveals three novel HLA-independent risk loci for ulcerative colitis," Gut, vol. 64, no. 4, pp. 571579, 2015.

[41] A. Aterido, J. D. Cañete, J. Tornero et al., "Genetic variation at the glycosaminoglycan metabolism pathway contributes to the risk of psoriatic arthritis but not psoriasis," Annals of the Rheumatic Diseases, vol. 78, no. 3, pp. 355-364, 2019.

[42] W. Gao, C. Sweeney, C. Walsh et al., "Notch signalling pathways mediate synovial angiogenesis in response to vascular endothelial growth factor and angiopoietin 2," Annals of the Rheumatic Diseases, vol. 72, no. 6, pp. 1080-1088, 2013.

[43] E. J. Woo, "Pearls: Simpson's paradox-understanding numbers that don't seem to make sense," Clinical Orthopaedics and Related Research, vol. 477, no. 11, pp. 2427-2428, 2019.

[44] B. Bayaraa, H. Arima, and S. Imafuku, "Body mass index in psoriatic patients with or without familial psoriasis," The Journal of Dermatology, vol. 47, no. 4, pp. 402-404, 2020.

[45] MAGIC, I. M. Heid, A. U. Jackson et al., "Meta-analysis identifies 13 new loci associated with waist-hip ratio and reveals sexual dimorphism in the genetic basis of fat distribution," Nature Genetics, vol. 42, no. 11, pp. 949-960, 2010. 